\title{
Correction of coronary endothelial dysfunction is a possible accessory mechanism for cellular therapy of the heart
}

\author{
Alexander S. Nemkov, Sergey. A. Belyi, Vladimir V. Komok, Oleg A. Grinenko, Nikolay S. Bunenkov \\ Department of Cardiovascular Surgery, First St.Petersburg State I. Pavlov Medical University, St.Petersburg, Russia
}

Professor Dr. Alexander S. Nemkov, Department of Cardiovascular Surgery, The $1^{\text {st }}$ St.Petersburg State I.Pavlov Medical University, L.Tolstoy St 6/8, 187089, St.Petersburg, Russia, St.Petersburg, Russia.
Phone: +7 (921) 7950047

E-mail: nemk_as@ mail.ru

\section{Summary}

Over 12 years of cellular therapy in cardiology, some dictinct positive results are obtained in randomized studies. However, exact mechanisms of hematopoietic stem cell actions are still unclear under these clinical conditions. Paracrine effects of cell therapy cannot explain all these effects. Enhanced neoangiogenesis upon stem cell injection is a proven mechanism for improvement of blood supply to the heart. Meanwhile, a decreased revascularization effect 3-4 years after cell therapy is followed by repeated myocardial improvement 6-9 mo after repeat- ed cell infusions with active development of collateral vessels, thus suggesting an additional mechanism for improvement of coronary blood supply. Restoration of regulatory functions of endothelium and smooth muscle cells, including increased NO synthase activity of endothelium and its interactions with myocardiocytes may represent a probable mechanism for this action.

\section{Keywords}

bone marrow cells, myocardium, cell therapy, endothelial dysfunction, mechanism of action.

\section{Introduction}

Precise mechanisms of cellular therapy of heart disorders with hematopoietic stem cells are unknown so far. However, they draw attention of clinicians and researchers, due to distinct positive effects observed in several studies. E.g., primary pre-clinical studies carried out in USA [9], and initial clinical studies in Düsseldorf (Germany) performed by Strauer et al. [13] and in Rostock by Steinhoff et al. [12] used autologous bone marrow cells (ABMC) for treatment of coronary artery disease (CAD). Trials with CAD patients have revealed a positive functional response of the cellular therapy. Over the last 12 years since introduction of this treatment modality, some distinct positive results are shown in randomized clin- ical studies. This treatment still has not become a standard of medication for CAD patients, despite of several meta-analyses $[1,5]$ have shown some benefits of the cellular therapy.

A general idea of possible paracrine effects produced by the cellular therapy cannot explain a variety of its actions and needs further interpretations. Intensive collateral formation (neoangiogenesis) in the areas of cell injections is a proven effect of cellular therapy. There are some effects, however, which cannot be explained solely by neoangiogenesis. To our mind, these effects represent indirect evidence for regeneration of endothelial lining in coronary arteries, including those of microcirculatory bed. Hence, the aim of our work was to provide an update of results confirming potential regenerative effects of intracoronary bone marrow cell infusions. 


\section{Material and methods}

Since 2003, a study at the St.Petersburg State I.Pavlov Medical University (SPBMU) has performed a controlled non-randomized open label study aimed for clinical evaluation of ABMC intracoronary infusions to ninety-seven patients with sufficiently affected coronary bed, however, being non-eligible for coronary bypass (CABG), or coronary angioplastics. The patients received intracoronary injections of ABMC obtained by multiple sternal and iliac punctures and isolated by a two-stage gradient centrifugation ( $900 \mathrm{~g}$ for 15 min followed by $700 \mathrm{~g}$ for $15 \mathrm{~min}$ ) using hydroxyethyl starch solution for separation. A total yield of nucleated cells per sample was $6.8 \pm 3.5 \times 10^{8}$ including $9 \pm 7.9 \times 10^{8}$ mononuclear cells, a mean number of CD34+ cells was $1.6 \pm 0.9 \times 10^{6}$. Cell viability was of myelokaryocytes was $95-98 \%$.

A comparison group consisted of thirty-seven patients who were not treated with ABMC. Coronary angiography was performed in all patients before ABMC injection. Heart visualization was performed by single photon emission computed tomography (SPECT) in 17 patients or positron emissive tomography (PET) examination in 10 patients before treatment and 1-2 years after ABMC injections, in order to assess local myocardial perfusion and metabolic rates.

\section{Results}

Clinical effect of AMBC injections was revealed in the study group, in terms of decreased anginal symptoms by one functional class $(68 \%)$, by two functional classes (12\%) whereas $20 \%$ of the patients did not feel any changes in their performance. Such effect was evident at 6 to 9 months after single AMBC injections. A similar long-term monitoring in control group did not reveal any changes in the functional class of angina pectoris. This positive effect of cellular therapy retains for about 3 years, followed by a decrease in antianginal effects at 4 to 5 years post-treatment $[2,7,11]$. Coronary angiography performed at these terms showed stable state of the coronary vessels, absence of additional atherosclerotic lesions and retained collateral circulation.

Our data on a distinct stepwise increase in myocardial perfusion within the $1^{\text {st }}$ year post-treatment also were confirmed by the results of PET examination with labeled ammonium. Improved myocardial metabolism in most myocardial segments was shown with fluordeoxyglucose (FDG) marker (Table.1). The most positive changes were noted for those segments with initially lesser capture of the radiolabeled drug. The same changes were found in a group of 10 patients (Fig. 1). An increase in these physiological parameters was detectable since 3 months after cellular therapy, followed by even more positive changes 6 and 12 mo after a single ABMC infusion $[8,11]$.

Table 1. PET data with FDG as a metabolic marker in patient M: dynamics of myocardial glucose consumption 12 mo post-treatment. Every of 17 segments of the heart had increased FDG marker, but the most positive changes was noted in those segments with initially lesser capture of radiolabeled drug (FDG)

\begin{tabular}{|l|l|l|l|}
\hline Segment & Before treatment (mmol/L/gram) & 12 Months after (mmol/L/gram) & Delta $\%$ \\
\hline 1 & 0,242 & 0,612 & +153 \\
\hline 2 & 0,315 & 0,642 & +104 \\
\hline 3 & 0,230 & 0,436 & +90 \\
\hline 4 & 0,277 & 0,604 & +118 \\
\hline 5 & 0,245 & 0,560 & +129 \\
\hline 6 & 0,110 & 0,359 & +226 \\
\hline 7 & 0,326 & 0,737 & +126 \\
\hline 8 & 0,310 & 0,602 & +94 \\
\hline 9 & 0,179 & 0,353 & +97 \\
\hline 10 & 0,249 & 0,479 & +92 \\
\hline 11 & 0,336 & 0,758 & +126 \\
\hline 12 & 0,245 & 0,678 & +177 \\
\hline 13 & 0,308 & 0,597 & +94 \\
\hline 14 & 0,197 & 0,385 & +95 \\
\hline 15 & 0,195 & 0,317 & +81 \\
\hline 16 & 0,301 & 0,632 & +107 \\
\hline 17 & 0,140 & 0,290 & +110 \\
\hline
\end{tabular}


Fig. 1. Mean myocardial consumption of glucose is increased after 12-mo follow-up in a group of 10 patients as shown by PET with FDG marker. The consumption index is increased by $57 \%(p=0.0001)$

One should note that improvement of myocardial perfusion was also traceable over wide myocardial areas by SPECT visualization, and, again, the most pronounced changes were seen for the segments with initially diminished capture of technetril-99mTc. Increased perfusion and higher metabolic recovery paralleled each other following intracoronary ABMC infusion. These radiological findings were accompanied by improved contractibility of the left ventricular myocardium, according to the echocardiography data. These positive changes were considered a result of improved collateral blood circulation $[8,11]$.
Box \& Whisker Plot

Var1 vs Var2

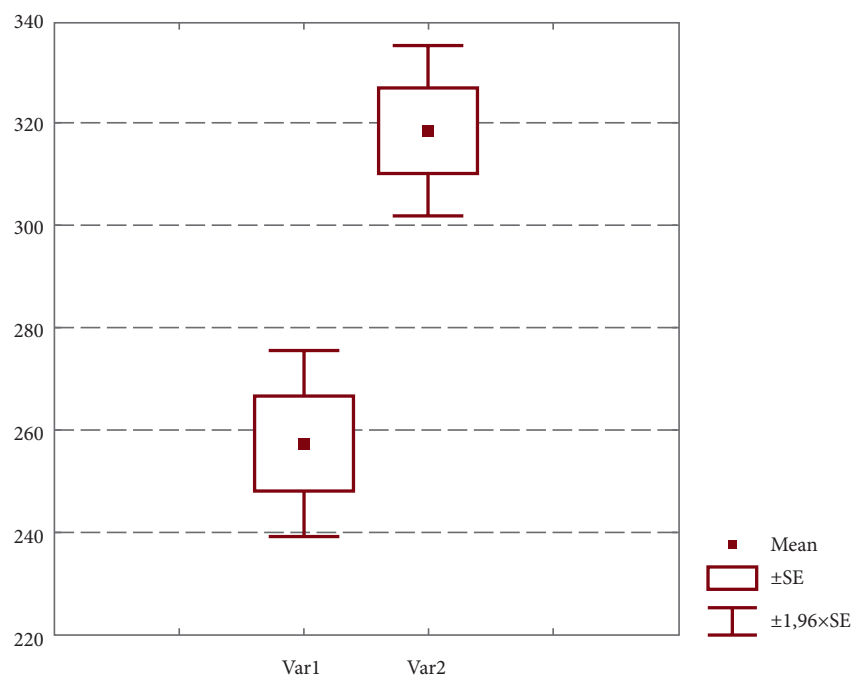

Moreover, our team has performed a longitudinal study of myocardial recovery in nineteen CAD patients subjected to intracoronary $\mathrm{ABMC}$ infusion. Serial SPECT evaluation performed for up to 3 years after the ABMC treatment has confirmed a decreased functional deficiency of the heart, thus suggesting an improved myocardial perfusion in the patients. The observed shrinkage of hypoperfused areas was case-dependent. Appropriate pro year changes were $0.79 \pm 9.7 \%$ to $5.4 \pm 8.7 \%$ of the total ischemic area $(<60 \%$ of normal values). The perfusion deficiency was increased four years later, thus indicating to worsening of blood supply (Fig. 2). It should be noted that repeated coronography did not, as a rule, exhibit a sufficient dynamics of initially affected coronary arteries (11).

Dynamic of median difference of myocardial proportion before and after ABMC at different time intervals (\%)

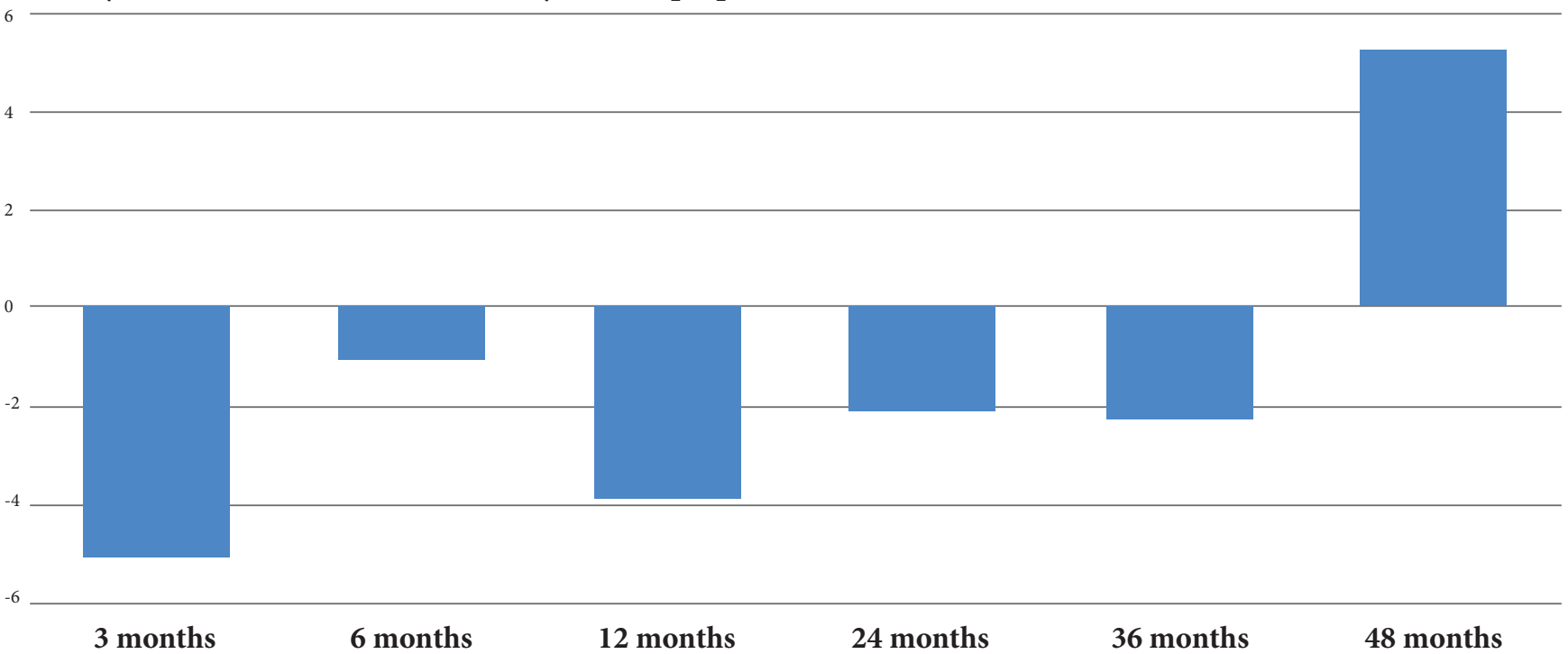

$\Delta$ median of myocardial proportion perfusion deficits

Fig. 2. Changes of myocardial areas with severe perfusion deficiency ( $>60 \%$, mean values) before treatment and at different time intervals after ABMC infusions, as assessed by SPECT technique in 17 patients. Decrease in perfusion deficiency was noted for 3 years, thus suggesting improvement of blood supply. Heart perfusion was again decreased 4 years later, as seen from suboptimal blood supply. 


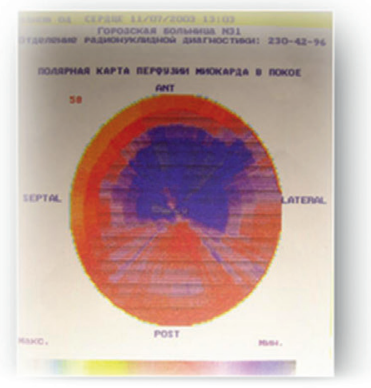

A

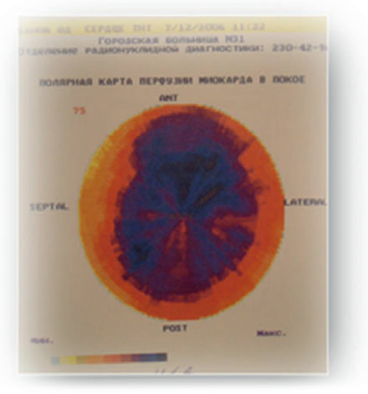

D

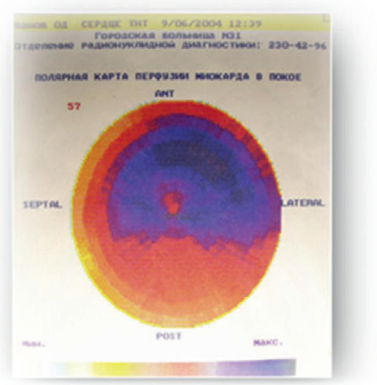

B

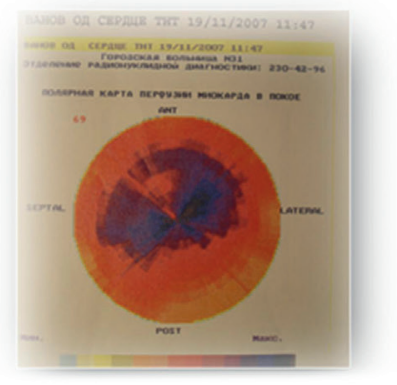

E

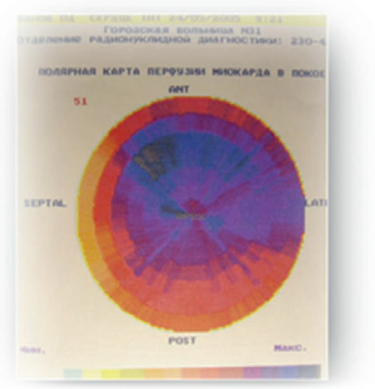

C

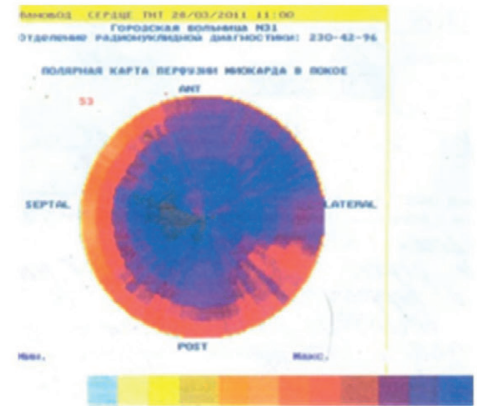

$\mathbf{F}$

Figure 3. Evaluation of myocardial perfusion by means of a single-photon computer tomography (SPECT) at the time before and 4 years after infusion of autologous bone marrow cells (ABMC), as well as 1 year after repeated ABMC injection to the coronary vessels. Blue color, good blood supply; white and yellow, deficient blood flow.

a.Before ABMC Perfusion deficiency, 18.4\%. Loading test, 75 Watt

b. 12 mo after ABMC. Perfusion deficiency, 8.7\%. Loading test, 100 Watt

c. 22 mo. Perfusion deficiency, $6.1 \%$. Loading test, 125 Watt

d. 39 mo. Perfusion deficiency, 6\%. Loading test, 125 Watt

e. 48 mo, Perfusion deficiency, 14\%. Loading test, 75 Watt

f. 60 mo (and 12 mo after repeated ABMC). Perfusion deficiency 6,8\%. Loading test 125 Watt

Repeated AMBC injections led to the secondary improvement of myocardial perfusion over a period of 6 to 12 months, as documented by SPECT assays with technetryl-99mTc (Fig. 3). A distinct therapeutic effect was also confirmed within 3 subsequent years, as evidenced by decrease in functional class of CAD.

\section{Discussion}

The mechanisms of collateral networks arising due to angiogenesis are discussed in the most works concerning potential effects of stem cells upon the ischemic myocardium. Some workers consider development of new collateral vessels among the main effects of cellular therapy. In most cases, the cytokine-mediated, or paracrine, effects seem to represent an immediate result of the cell therapy, i.e., the injected cells may be cytokine suppliers which provide an augmented local regeneration. One should note that the infused stem cells are active producers of growth factors boosting enhanced regeneration. The injected cells are, however, prone to subsequent death, due to their damage upon isolation and in vitro manipulations, as well as depletion of growth factors. In such instance, favorable effects of the cellular therapy should be, generally, limited by 3 to 6 months, being ascribed to the cytokine effects. In most cases, however, duration of the positive changes may be as long as 3 to 4 years, thus suggesting participation of an intrinsic regeneration component, e.g., an increased activity of resident cardiac stem cells [6].

Alternative hypotheses concerning de novo cardiomyocyte production are proposed by several workers. At the present time, some data are obtained in favor of myocardial cell renewal which, however, proceeds at slow rates [3]. The most convincing arguments for the cell regenerative component are presented by Quaini et al. who studied cellular chimerism in transplanted heart in patients with severe heart failure [10]. The authors transplanted female hearts to the male recipients, and a sufficient amount of male ( $\mathrm{Y}$ chromosome-positive) cells was detected in grafts of female origin, thus suggesting some cellular regenerative mechanisms 
following the heart transplantation. The mean ratio of male cells in female allografts was, respectively, $18 \%$ for myocardiocytes; $20 \%$ among vascular smooth muscle cells, and $14 \%$, for capillary endotheliocytes. The samples were taken at the $\mathrm{D}+4$ to $\mathrm{D}+552$ post-transplant . This result suggests an intensive blood-born homing of some male cells to the female heart transplant. Appropriate precursor cells may be hematopoietic, or endothelial by their origin. Hence, it should be noted that different cell populations are subject to renewal, i.e., cardiomyocytes, vascular smooth muscle cells, and capillary endotheliocytes which may expand in the cardiac graft. One may suggest that similar cells could regenerate in autologous heart of the patient with cardiovascular disorders, presuming the same regenerative pathways.

Additional data about potential intrinsic cell substitution, along with cytokine actions of the injected stem cells are presented by Dimmeler et al [15] in murine experiments. This model included trials with induced death of suicide gene-containing cells after their introduction to the myocardial syncytium, vascular endothelium, and vascular walls. In a model of acute myocardial infarction, the injected bone marrow cells were committed for endothelial differentiation, thus causing a sufficient improvement of myocardial contractibility. Induced cell death of the endothelium-committed cells was associated with decreased cardiac efflux. Interestingly, an induced death of the myocardium-committed cells did not exert such an effect. Moreover, elimination of NO synthase-expressing endothelial cells was associated with a decrease in capillary and arteriolar density. These data reflect potential effects of endothelial population upon functional ability of myocardium.

The endotheliocyte layer regulates transfer of stem cells through the vascular barrier via increased expression of adhesion molecules at the endothelium surface. The cells with different phenotypes exhibit different ability for transendothelial migration. One may suggest that reconstitution of endothelial function, including the cellular transport functions, may play a sufficient role in cardiac regeneration.

The first experimental work suggesting a mechanism of bone marrow stem cell effects upon endothelial dysfunction was presented by the workers from the USA [14]. Using an animal model of atherosclerosis-prone mice, they demonstrated that treatment with bone marrow endothelial precursors caused enhancement of NO production by endothelial cells, along with increase of endothelium-dependent vascular relaxation and decreased thickness of lipid plaques. This finding allows to suggest that reconstution of endothelial dysfunction with bone marrow cells may be indispensable for effective atherosclerosis treatment, both by correction of endothelial dysfunction, and prevention of lipid plaque development.

Functional and morphological interrelations between coronary endotheliocytes and cardiomyocytes are quite complex, multifaceted, being mediated not only by NO, but also by a number of other substances produced by endothelial cells which, in turn, may actively modify the cardiomyocyte functions [4]. Therefore, a correction of endothelial function in coronary vessels may be among future tasks for cellular therapy, being quite important for treatment of myocardial heart insufficiency.

\section{Conclusion}

Intracoronary injections of $\mathrm{ABMC}$ in $\mathrm{CAD}$ patients are accompanied by increased radiomarker capture over the entire myocardium, including the segments with satisfactory blood flow and hypoperfused areas, thus suggesting a diffuse mechanism for improved blood circulation and heart metabolism. Gradual fading of clinical and radiological improvement in cardiac blood supply is observed 3 to 4 years after cellular therapy, despite retained collateral flow and stabilization of atherosclerotic coronary damage. Subsequent improvement in blood supply resulting from repeated ABMC injections argues for additional, probably functional, mechanisms of increased blood supply associated with the cellular therapy.

This supplementary mechanism of cell therapy upon intracoronary ABMC injection may be potentially based on correction of endothelial dysfunction in coronary arterioles and capillary bed. The NO-dependent relaxation of coronary arterioles is connected with smooth muscle cell functions which may regenerate following the cellular therapy. This mode of correction is presumed to occur due to hematopoietic or endothelial precursors.

A renewal of endothelial or smooth muscle cells at the account of the bone marrow cells may be a sufficient component of restored functioning of a blood flow regulation, i.e., via endothelial production of $\mathrm{NO}$ and other substances which are able to modify the interactions between endothelium and cardiomyocytes.

Renewal rates of endotheliocytes in the cardiac vessels may proceed more rapidly that among cardiomyocytes, being, however, much slower than regeneration of blood cells. Therefore, the effects of restored endothelium cannot be expected earlier than several months after intracoronary ABMC injection. Therefore, a probable duration of appropriate effect, as seen from time dynamics of clinical and radiological signs after ABMC treatment, may be as long as 3 to 4 years. A distinct therapeutic effect was also confirmed by decrease in CAD functional class, thus allowing to recommend intracoronary AMBC injections every 3-4 years. We need, however, additional studies, in order to test our hypothesis concerning restoration of local endothelial function, and influence of this process upon the cardiomyocyte function.

\section{Conflict of interest}

None declared

\section{References}

1. Abdel-Latif A, Bolli R, Tleyjeh IM, et al. Adult bone marrow-derived cells for cardiac repair. A systematic review and meta-analysis. Arch Intern Med 2007; 167(10):989-997.

2. Burnos SN, Nemkov AS, Belyŭ SA, Lukashenko VI. Ejection fraction and sizes of the left ventricle of the heart after intracoronary administration of autologous mononuclear cells of the bone marrow in patients with coronary artery 
disease with low ejection fraction.// Vestn Khir Im I I Grek 2011;170(4):16-19. (In Russian)

3. Leri A, Kajstura J, Anversa P. Mechanisms of myocardial regeneration. Trends Cardiovasc Med 2011; 21(2):52-58.

4. Lynn LS, Lam CSP, Segers VFM, Brutsaert DL, De Keulenaer GW. Cardiac endothelium-myocyte interaction: clinical opportunities for new heart failure therapies regardless of ejection fraction. Eur Heart J 2015; doi:10.1093/eurheartj/ ehv132

5. Jeevanantham V, Butler M, Saad A, et al. Adult bone marrow cell therapy improves survival and induces long-term improvement in cardiac parameters : a systematic review and meta-analysis. Circulation 2012;126:551-568

6. Marban E, Cheng Ke. Heart to heart: the elusive mechanism of cell therapy. Circulation 2010; 121:1981-1984.

7. Nemkov AS, Belyĭ SA, Nesteruk IuA, et al. Quality of life in patients with coronary artery disease after stem cell therapy.Vestn Khir Im I I Grek. 2012;171(1):16-20. (In Russian).

8. Nesteruk JA,Nemkov AS, Beliy SA, et al. Evaluation of myocardial blood supply and methabolism after autologous bone marrow mononuclear cells intracoronary infusion. Regional Blood Supply and Microcirculation 2014; 13(3):2330. (In Russian)

9. Orlic D, Kajstura J, Chimenti S, Jakoniuk I, Anderson SM, Li B, Pickel J, McKay R, Nadal-Ginard B, Bodine DM, Leri A, Anversa $\mathrm{P}$. Bone marrow cells regenerate infarcted myocardium. Nature 2001; 410:701-705.
10. Quaini F, Urbanek K, Beltrami AP, Finato N, Beltrami CA, Nadal-Ginard B, Kajstura J, Leri A, Anversa P. Chimerism of the Transplanted Heart. N Engl J Med 2002; 346:5-15.

11. Sedov VM, Burnos SN, Nemkov AS, et al. Changes of myocardial perfusion after intracoronary administration of autologous bone marrow mononuclear cells in patients with coronary artery disease. Five-year follow-up. Regional Blood Supply and Microcirculation 2011; 10(2):19-23. (In Russian).

12. Stamm C, Westphal B, Kleine HD, Petzsch M, Kittner C, Klinge H, Schümichen C, Nienaber CA, Freund M, Steinhoff G. Autologous bone-marrow stem-cell transplantation for myocardial regeneration. Lancet 2003; 361(9351):45-46.

13. Strauer BE, Brehm M, Zeus T, Kostering M, Hernan$\operatorname{dez}$ A, Sorg RV, Kogler G, Wernet P. Repair of infarcted myocardium by autologous intracoronary mononuclear bone marrow cell transplantation in humans. Circulation 2002;106:1913-1918.

14. Yao L, Heuser-Baker J, Herlea Pana O, et al. Bone marrow endothelial progenitors augment atherosclerotic plaque regression in a mouse model of plasma lipid lowering. Stem Cells 2012; 30(12): 2720-2731.

15. Yoon Ch-H, Koyanagi M, Iekushi K, Seeger F, Urbich C, Zeiher A, Dimmeler S. Mechanism of improved cardiac function after bone marrow mononuclear cell therapy. Role of cardiovascular lineage commitment. Circulation 2010;121:2001-2020. 